\title{
DESCRIPTIVO Y ANÁLISIS DE LAS RADIACIONES IONIZANTES EN LA CIUDAD DE TACNA
}

Responsable: MSc.José Antonio Tiburcio Moreno Miembro: Lic.Jesus Alfredo Chacaltana Garcia Miembro: Bach.Fanny Esmeralda Mori Escobar

\section{RESUMEN}

En este artículo se analiza, de manera cualitativa, el impacto sobre las personas que de manera directa o indirecta se encuentran expuestas, de manera involuntaria, a altas dosis de exposición a radiaciones del tipo ionizante; debido a que en muchos centros médicos de la ciudad de Tacna se usa este tipo de radiaciones como método de diagnostico de algunas enfermedades, sin tener en cuenta los protocolos de seguridad para el manejo de equipos que trabajan con sustancias radiactivas y que generan este tipo de radiaciones, las cuales, al interaccionar con organismos vivos, pueden causar potencialmente una serie de enfermedades por la alta dosis de exposición a ellas.

\section{ABSTRACT}

In this paper, the impact is analyzed of qualitative way on the people who of direct or indirect way are exposed, of involuntary way, to high doses of exhibition to radiations of the ionizing type, due to that in many medical centers of the city of Tacna they are used this type of radiations as method of diagnose some diseases, without considering the protocols of security for the handling of equipment that works with radioactive substances and that it generates east type of radiations, which when interacting with alive organisms can potentially cause a series of diseases by the high dose of exhibition them. calidad.

Palabras claves: Radiación ionizante, radiodiagnóstico, radiografia dental, dosis medias de radiación, control de

\section{INTRODUCCIÓN}

La radiación ionizante está en todas partes. Llega desde el espacio exterior en forma de rayos cósmicos. Está en el aire en forma de emisiones del radón radiactivo y su progenie. Los isótopos radiactivos que se originan de forma natural entran y permanecen en todos los seres vivos. Es inevitable. De hecho, todas las especies de este planeta han evolucionado en presencia de la radiación ionizante. Aunque los seres humanos expuestos a dosis pequeñas de radiación pueden no presentar de inmediato ningún efecto biológico aparente, no hay duda de que la radiación ionizante, cuando se administra en cantidades suficientes, puede causar daños. El tipo y el grado de estos efectos son bien conocidos[1]. Si bien la radiación ionizante puede ser perjudicial, también tiene muchas aplicaciones beneficiosas. El uranio radiactivo genera electricidad en centrales nucleares instaladas en muchos paises. En medicina, los rayos $X$ permiten obtener radiografías para el diagnóstico de lesiones y enfermedades internas. Los médicos especializados en medicina nuclear utilizan material radiactivo como trazadores para formar imágenes detalladas de estructuras internas y estudiar el metabolismo[2]. En la actualidad se dispone de radio fármacos terapéuticos para tratar trastornos como el hipertiroidismo y el cáncer. Los médicos utilizan en radioterapia rayos gamma, haces de electrones, neutrones y otros tipos de radiación para tratar el cáncer. Los ingenieros emplean material radiactivo en las operaciones de registro de pozos petroliferos y para medir la densidad de la humedad en los suelos. Los radiólogos industriales se valen de rayos $X$ en el control de calidad para observar las estructuras internas de aparatos fabricados. Las señales de las salidas de edificios y aviones contienen tritio radiactivo para que brillen en la oscuridad en caso de fallo de la energía eléctrica. Estos numerosos usos de la radiación ionizante y de los materiales radiactivos mejoran la calidad de vida y ayudan a la sociedad de muchas maneras. Pero siempre se deben sopesar los beneficios de cada uso con sus riesgos. Estos pueden afectar a las personas que intervienen directa o indirectamente en la aplicación de la radiación o el material radiactivo[3] y al medio ambiente. En tal sentido, los beneficios siempre deben superar a los riesgos cuando se trate de utilizar la radiación ionizante.

\section{CONCEPTOS PRELIMINARES}

\section{a) Radiación ionizante:}

La radiación ionizante consiste en particulas, incluidos los fotones, que causan la separación de electrones de átomos y moléculas. Algunos tipos de radiación de energía relativamente baja, como la luz ultravioleta, sólo pueden originar ionización en determinadas circunstancias[4]. Para distinguir estos tipos de radiación, se establece un límite energético inferior arbitrario para la radiación ionizante, que se suele situar en torno a los 10 kilos electronvoltios (keV). La radiación ionizante directa consta de partículas cargadas, que son los electrones energéticos, los positrones, los protones, las partículas alfa, 
los mesones cargados, los muones y los iones pesados (átomos ionizados). Los tipos más comunes de radiación ionizante indirecta son los generados por fotones con energia superior a $10 \mathrm{keV}$ (rayos $\mathrm{X}$ y rayos gamma) y todos los neutrones. Los fotones de los rayos $X$ y gamma interactúan con la materia y causan ionización de tres maneras diferentes como mínimo:

1. Los fotones de energía más baja interactúan sobre todo mediante el efecto fotoeléctrico, por el que el fotón cede toda su energía a un electrón, que entonces abandona el átomo o molécula.

2. Los fotones de energía intermedia interactúan fundamentalmente mediante el efecto Compton, en virtud del cual el fotón y un electrón colisionan esencialmente como partículas. El fotón continúa su trayectoria en una nueva dirección con su energía disminuida, mientras que el electrón liberado parte con el resto de la energía entrante.

3. La producción de pares sólo es posible con fotones cuya energía sea superior a $1,02 \mathrm{MeV}$. El fotón desaparece, y en su lugar aparece una pareja electrónpositrón. Estos electrones y positrones energéticos se comportan entonces como radiación ionizante directa.

\section{b) Velocidad de Desintegración:}

Es conocido que los nucleidos radiactivos se desintegran espontáneamente[5] con una velocidad determinada y constante, que depende de la naturaleza del nucleído. El núcleo inestable emite una partícula (o radiación) característica, transformándose de este modo en un núcleo diferente, que también puede ser, o no, radiactivo. A medida que se van produciendo, los átomos de un determinado radioisótopo de una masa de sustancia radiactiva irán disminuyendo, llegando un momento en que su número se haya reducido a la mitad. A ese período de tiempo se le llama período de semidesintegración y al igual que la velocidad de desintegración es característico de cada nucleído.

Los períodos de semidesintegración para las distintas sustancias radiactivas varian desde la millonésima de segundo hasta millones de años[3]. Tal como podemos apreciar en la tabla 1.
TABLA 1: Disminución de la Actividad Radiactiva con el Tiempo.

\begin{tabular}{|c|c|c|c|c|c|c|}
\hline \multirow{2}{*}{ Elemento } & \multirow{2}{*}{ Periodo } & \multicolumn{5}{|c|}{$\begin{array}{l}\text { Número por el que hay que Multiplicarse la Actividad } \\
\text { para saber lo que queda después de: }\end{array}$} \\
\hline & & 1 Día & $\begin{array}{c}1 \\
\text { Semana }\end{array}$ & $1 \mathrm{mes}$ & 3 Meses & 1 Año \\
\hline $\begin{array}{c}\text { Tecnecio } \\
\text { Tc-99 } \\
\end{array}$ & 6 horas & 0,063 & 0,000 & 0,000 & 0,000 & 0,000 \\
\hline |odol. & 8 dias & 0,917 & 0,545 & 0,074 & 0,000 & 0,000 \\
\hline $\begin{array}{c}1311 . \\
25\end{array}$ & 60 dias & $0, \$ 89$ & 0,922 & 0,707 & 0,353 & 0,015 \\
\hline $\begin{array}{l}\text { Iridio } \\
\text { Ir - } 192\end{array}$ & 74 dias & 0,991 & 0,937 & 0,755 & 0,442 & 0,033 \\
\hline $\begin{array}{l}\text { Cobalto } \\
\text { Co } .60\end{array}$ & 5,3 años & 1,000 & 1,000 & 1,000 & 0,968 & 0,877 \\
\hline $\begin{array}{l}\text { Cesio } \\
\text { Cs }-137\end{array}$ & 30 años & 1,000 & 1,000 & 1,000 & 1,000 & 0,977 \\
\hline $\begin{array}{l}\text { Tritio } \\
\mathrm{H}-3\end{array}$ & 12,3 años & 1,000 & 1,000 & 1,000 & 1,000 & 0,945 \\
\hline $\begin{array}{c}\text { Carbono } \\
C \quad-14\end{array}$ & 5600 años & \multicolumn{5}{|c|}{$\begin{array}{l}\text { Su actividad se puede considerar constante incluso } \\
\text { después de muchos años }\end{array}$} \\
\hline
\end{tabular}

\section{RESULTADOS OBSERVADOS EN LA CIUDAD DE TACNA}

Se puede observar que en las avenidas principales de la ciudad de Tacna (Bolognesi, Patricio Meléndez, San Martin y otras), se encuentran instalados una gran cantidad de consultorios médicos, principalmente de la especialidad de odontología y otros que se dedican al diagnostico de algunas enfermedades mediante imágenes (radiografías), utilizando generalmente rayos $\mathrm{X}$, los cuales son radiaciones de naturaleza electromagnética altamente energéticas que pueden atravesar fácilmente los muros de estas instalaciones precariamente utilizadas como consultorios, las cuales no han sido diseñadas para brindar este tipo de servicios. Sin embargo, se manipulan equipos con fuentes radioactivas, permitiendo que interaccionen con el medio que las rodea. Para dar una idea de las dosis a las que potencialmente estarian expuestas las personas directa 0 indirectamente, establecemos los siguientes conceptos necesarios para valorar la relación causa-efecto que causa estas interacciones:

a) Actividad, de un radio nucleido se define en un instante determinado y desde un estado de energía definido como el número de transiciones espontáneas, o lo que es lo mismo, la intensidad con la que se desintegra un material radiactivo y cuya unidad de medida en el sistema internacional es el becquerel $(\mathrm{Bq})$. También se utiliza el curio $(\mathrm{Ci})$ $(1 \mathrm{Ci}=37 \mathrm{Gbq})$;

b) Dosis Absorbida, que es la cantidad de energia absorbida por unidad de masa de 
material irradiado y su unidad de medida es el Gray (Gy);

c) Exposición, que se emplea para medir la capacidad de la radiación para producir iones en el aire. Su unidad de medida es $\mathrm{C} / \mathrm{Kg}$ (el Roentgen ( $R$ ) es una magnitud que también mide la exposición, aunque actualmente ha caido en desuso).

d) Dosis Efectiva, que se emplea para representar el efecto de las radiaciones sobre el ser humano en su conjunto. Tiene en cuenta tanto el tipo de radiación de que se trata como los órganos del cuerpo afectados. La unidad de medida en el sistema internacional es el sievert (Sv), otra unidad de medida que tradicionalmente se ha utilizado es el rem (equivalencia: $1 \mathrm{rem}=10 \mathrm{mSv}$ ), aunque cada vez es menos habitual su uso.

La Tabla 2 muestra de manera resumida las unidades radiológicas y las equivalencias existentes entre ellas;

TABLA 2: Unidades Radiológicas y Equivalencias

\begin{tabular}{|c|c|c|c|}
\hline \multicolumn{2}{|c|}{$\begin{array}{l}\text { Unidades Radiológicas Sistema } \\
\text { Internacional (SI) }\end{array}$} & \multirow[b]{2}{*}{$\begin{array}{l}\text { En otras } \\
\text { Unidades SI }\end{array}$} & \multirow[b]{2}{*}{$\begin{array}{l}\text { Unidad especial } \\
\text { antigua }\end{array}$} \\
\hline $\begin{array}{l}\text { Magnitud } \\
\text { y Simbolo }\end{array}$ & $\begin{array}{l}\text { Nombre Especial } \\
\text { y Simbolo (SI) }\end{array}$ & & \\
\hline Exposición $(X)$ & - & $\mathrm{C} / \mathrm{Kg}$ & Roentgen (R) \\
\hline $\begin{array}{l}\text { Dosis Absorbida } \\
\text { (D) }\end{array}$ & Gray (Gy) & $\mathrm{J} / \mathrm{Kg}$ & $\operatorname{Rad}(\mathrm{rad})$ \\
\hline $\begin{array}{c}\text { Dosis Efectiva } \\
\text { (H) }\end{array}$ & Sievert (Sv) & $\mathrm{J} / \mathrm{kg}$ & Rem (rem) \\
\hline Actividad (A) & Becquerelio (Bq) & a- 1 & Curio (Ci) \\
\hline \multicolumn{4}{|c|}{ Equivalencias } \\
\hline \multicolumn{2}{|c|}{$1 \mathrm{R}=2,58 \times 10^{-4} \mathrm{C} / \mathrm{Kg}$} & \multicolumn{2}{|c|}{$1 \mathrm{C} / \mathrm{Kg}=3876 \mathrm{R}$} \\
\hline \multicolumn{2}{|c|}{$1 \mathrm{rad}=1 \times 10^{-2} \mathrm{~Gy}=1 \mathrm{cG}$} & \multicolumn{2}{|c|}{$1 \mathrm{~Gy}=100 \mathrm{rad}$} \\
\hline \multicolumn{2}{|c|}{$1 \mathrm{rem}=1 \times 10^{-2} \mathrm{~Sv}=1 \mathrm{cSv}$} & \multicolumn{2}{|c|}{$1 \mathrm{~Sv}=100 \mathrm{rem}$} \\
\hline \multicolumn{2}{|c|}{$1 \mathrm{Ci}=3,7 \times 1010 \mathrm{~Bq}=37 \mathrm{GBq}$} & \multicolumn{2}{|c|}{$1 \mathrm{~Bq}=2,70 \times 10^{-11} \mathrm{Ci}=27 \mathrm{pCi}$} \\
\hline
\end{tabular}

El comité científico de las Naciones Unidas para el Estudio de los Efectos de las Radiaciones Atómicas $^{[1]}$ (UNSCEAR) estima regularmente las dosis debidas a las fuentes naturales y estableció un valor mundial medio de 2,4 mSv, esta dosis recibida se conoce como radiación de fondo para un habitante adulto y está repartida según muestra la Figura 1.

Total 2,4 mSv

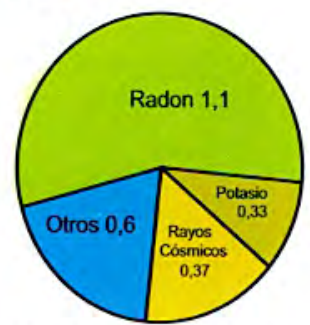

FIGURA. Dosis de radiación debida a Fuentes naturales
Según datos publicados recientemente ${ }^{[6,7]}$ una persona expuesta a una radiografía dental aproximadamente absorbe $0,10 \mathrm{mSv}$. La dosis de radiación efectiva de este procedimiento es aproximadamente la misma cantidad que una persona en promedio recibe de radiación de fondo en 10 dias, tal como se puede apreciar en la Tabla 3. En paises de la Unión Europea, por ejemplo España, se estima que se realizan un promedio de 90 exploraciones dentales por cada 1000 habitantes. Se estima que el radiodiagnóstico dental contribuye con una extensa parte a la dosis colectiva anual global sobre la población mundial con aproximadamente 14 $000 \mathrm{~Sv} /$ hombre, que equivale a $0,002 \mathrm{mSv} /$ persona.

A continuación se muestra una comparación de las dosis de radiación efectivas con la exposición de fondo para varios procedimientos radiológicos:

TABLA 3: Comparación de Procedimientos Radiológicos y la Radiación de Fondo

\begin{tabular}{|l|c|c|}
\hline \multicolumn{1}{|c|}{ Procedimiento } & $\begin{array}{c}\text { Dosis de Radiación } \\
\text { Efectiva }\end{array}$ & $\begin{array}{c}\text { Comparable con la } \\
\text { Radiación Natural de } \\
\text { Fondo durante: }\end{array}$ \\
\hline Región abdominal: & $10 \mathrm{mSv}$ & 3 años \\
\hline $\begin{array}{l}\text { Tomografia axial } \\
\text { computarizada (TAC) - Cuerpo }\end{array}$ & $1,6 \mathrm{mSv}$ & 6 meses \\
\hline Pielograma intravenoso (PIV) & $4 \mathrm{mSv}$ & 16 meses \\
\hline $\begin{array}{l}\text { Radiografia - Tracto digestivo } \\
\text { inferior }\end{array}$ & $2 \mathrm{mSv}$ & 8 meses \\
\hline $\begin{array}{l}\text { Radiografia - } \\
\text { Tracto digestivo alto }\end{array}$ & $10 \mathrm{mSv}$ & 3 meses \\
\hline $\begin{array}{l}\text { Tomografia axial } \\
\text { computarizada (TAC) Abdomen }\end{array}$ & & 3 años \\
\hline $\begin{array}{l}\text { Tomografia } \\
\text { computarizada (TAC) Cabeza }\end{array}$ & & \\
\hline Torax: & & \\
\hline Radiografia de tórax & & \\
\hline
\end{tabular}

Como puede observarse en la Tabla 3, en estos procedimientos radiológicos de intervención que usan rayos $X$, el nivel de riesgo depende del procedimiento porque algunos usan muy poca radiación; pero otros, más complejos, usan mucha más. En general, el riesgo de enfermedades debe compararse con los beneficios del procedimiento. Por ejemplo, los usados para eliminar una obstrucción en un vaso sanguíneo, reparar un área de debilidad en un vaso dilatado, o desviar el flujo sanguineo de malformaciones vasculares, usan más radiación. Sin embargo, estos complejos procedimientos a menudo pueden salvarle la vida al paciente y los riesgos asociados con la radiación son una consideración secundaria. 
En nuestra ciudad, gran cantidad de consultas odontológicas diarias simultáneamente se llevan a cabo, teniendo en cuenta que la radiación ionizante puede propagarse fácilmente en todas las direcciones posibles, como lo hace una onda electromagnética; podemos establecer que gran cantidad de personas están expuestas potencialmente a dosis elevadas de este tipo de radiaciones, porque no se toman en cuenta todos los medios de seguridad y blindaje para este tipo de equipos que usan material radiactivo. En nuestro estudio, se realizó un censo ${ }^{[3]}$, cuyo resumen se muestra en la Tabla 4, de los principales centros médicos que cuentan estos equipos. En muchos casos, no contamos con la colaboración de los responsables de estas instalaciones. Asimismo, no se tuvo acceso al registro diario oficial de las consultas que se realizan para poder realizar estimaciones estadisticas de consultas realizadas a lo largo de todo el año, debido a que muchos de estos consultorios actúan casi de manera informal.

Tabla 4: Número de Atenciones Para Diferentes Especialidades Médicas

\begin{tabular}{|c|c|c|c|l|l|}
\hline $\begin{array}{c}\text { Especialidad } \\
\text { Medica }\end{array}$ & $\begin{array}{c}\text { Múmero de } \\
\text { Consultorios }\end{array}$ & $\begin{array}{c}\text { Promedio } \\
\text { de Atenciones } \\
\text { Mensuales }\end{array}$ & $\begin{array}{c}\text { Consultorios } \\
\text { con: RX, } \\
\text { Eecogrados, etc. }\end{array}$ & $\begin{array}{c}\text { Seguridad } \\
\text { Radiologica }\end{array}$ & $\begin{array}{c}\text { Estado de } \\
\text { los Equipos }\end{array}$ \\
\hline Odontologia & 20 & 450 & 16 & Escass & Obsoletos \\
\hline Ottalmologia & 12 & 160 & 08 & & Regular \\
\hline Otras Espec. & 18 & 120 & & & \\
\hline
\end{tabular}

Se puede apreciar en esta tabla, la gran cantidad de consultas exploratorias dentales mensuales realizadas, en la mayoria de estos casos, según se indicó en el sondeo, se aplica radiodiagnóstico en alrededor del $85 \%$ de los casos.

En cuanto a las medidas de protección, indicaron utilizar mandiles especiales, los cuales en ninguno de los casos fueron mostrados. Asimismo, en algunos de estos centros médicos que emplean diagnostico mediante Rayos-X, manifestaron utilizar biombos de metal, blindaje en los muros y cámaras especiales; sin embargo, en la exploración visual realizada no se pudo apreciar estos medios de protección; por el contrario, los muros, techos y pisos correspondian a una oficina normal. Respecto a los equipos se notó obsolescencia en la mayoria de los casos.

\section{CONCLUSIÓN}

Parece evidente que debido al número excesivo de exploraciones dentales que se vienen realizando diariamente en nuestra ciudad con la consiguiente gran cantidad de radiografias dentales practicadas, se tiene una situación sumamente peligrosa en cuanto a la dosis de radiación ionizante a las que estaría sometido los pacientes y publico en general. Esta situación puede ser atribuible a que dichas exploraciones radiológicas se efectúan con equipos anticuados, con averias, técnicas obsoletas y alteraciones o manejo incorrecto en la técnica de revelado radiográfico. Bajo estas circunstancias, somos de la opinión que las autoridades competentes: Ministerio de Salud, Municipalidad Provincial de Tacna, Región Tacna, etc., realicen una auditoria y evaluación real de la situación en las cuales se encuentran estos equipos radiológicos y verificar las técnicas de revelado utilizadas en los diferentes centros médicos de la ciudad que prestan este tipo de servicios. Es necesario, además, poner en práctica el D.S. №09-97-EM sobre la Reglamentación de Seguridad Radiológica y otras normas vigentes sobre control de calidad para el uso de material radiactivo, lo cual debe ser de entera responsabilidad de los titulares de estos establecimientos médicos, para que se eliminen esta serie de dificultades que producen aumento en las dosis administradas a los pacientes. Asimismo, se debe certificar que todas estas instalaciones donde se llevan a cabo estas prácticas medicas deben poseer algún tipo de material de protección personal, como son: delantales plomados y collarín tiroideo, para la disminución de radiación en radiología intraoral no solo para el paciente sino hacia el propio profesional que como ya se ha observado maneja dosis individuales relativamente elevadas.

\section{BIBLIOGRAFÍA}

1.... Sources and Effects of lonizing Radiation. United Nations Scientific Committee on the Effects of Atomic Radiation UNSCEAR 2000 Report to the General Assembly, with Scientific Annexes. Volume: Sources. New York, United Nations 2000

2. Hall, EJ. 1994. Radiobiology for the Radiologist. Filadelfia: JB Lippincott.

3. .J. Tiburcio M., J. Chacaltana G., F. Mori E., Análisis Descriptivo de las Radiaciones Ionizantes en la ciudad de Tacna, Proyecto de Investigación (R.F. 3136-2005-FACl), COIN, UNJBG, 2005.

4. Goodhead, DJ.,1988, Spatial and temporal distribution of energy. Health Phys 55:231-240.

5. Mettler, FA, AC Upton. 1995. Medical Effects of lonizing Radiation. Nueva York: Grune \& Stratton.

6. Alcaraz M, Martínez-Beneyto $\mathrm{Y}$, Velasco $\mathrm{E}$., Control de calidad en instalaciones de radiodiagnóstico dental. Revista Europea de Odontoestomatologia, 1999; 11: 265-74.

7. Jodar, S., Alcaraz, M., Martinez-Beneyto, y., Manejo de las radiaciones ionizantes en instalaciones dentales españolas: intraorales $y$ panorámicos, Av. Odontoestomatol, ene.-feb. 2005, vol.21, no.1, p.361-370. ISSN 0213-1285. 